\title{
Talleres y evaluaciones de proceso: una herramienta que mejora el rendimiento en asignaturas integradas
}

\section{Workshops and Process Evaluations: Tools that Improve Performance in Integrated Subjects}

\author{
Workshops e avaliações de processo: uma \\ ferramenta que melhora o rendimento em \\ disciplinas integradas
}

Gabriela Urrejola Contreras, MSc ${ }^{1 *}$

Paulina Tenore Venegas, $\mathrm{MSc}^{1}$

Sebastián Elgueta Perinni, MSc ${ }^{1}$

Miguel Pérez Lizama, $\mathrm{PhD}^{1}$

Paula Araya Herrera, $\mathrm{MSc}^{2}$

Pía Daniela Campos Reinoso, $\mathrm{MSc}^{3}$

Recibido: 18 de noviembre de 2019 · Aceptado: 13 de marzo de 2020

Doi: https://doi.org/10.12804/revistas.urosario.edu.co/revsalud/a.9276

Para citar este artículo: Urrejola Contreras G, Tenore Venegas P, Elgueta Perinni S, Pérez Lizama M, Araya Herrera P, Campos Reinoso PD. Talleres y evaluaciones de proceso: una herramienta que mejora el rendimiento en asignaturas integradas. Rev Cienc Salud.

2020;18(2):1-15. https://doi.org/10.12804/revistas.urosario.edu.co/revsalud/a.9276

\section{Resumen}

Introducción: una tendencia actual en la educación superior es fusionar materias y articular las asignaturas integradas; sin embargo, hay menor rendimiento y un incremento de la reprobación. Se ha descrito como favorable la aplicación de evaluaciones de procesos como instrumentos de enseñanza y aprendizaje en ciencias de la salud, criterio pertinente de considerar en asignaturas de gran volumen de contenidos. El objetivo de este trabajo fue valorar el efecto de la evaluación del proceso sobre una asignatura integrada de anatomía y fisiología en estudiantes de ciencias de la salud y su relación con el rendimiento. Materiales y métodos: estudio experimental con 144 estudiantes de la Escuela de Ciencias de la Salud en la asignatura de Estructura y Función, distribuidos en dos grupos: control y experimental. Se diseñó e implementó una intervención metodológica para evaluar su efecto en el rendimiento académico. Resultados: la intervención del proceso en el grupo control experimental mejoró el rendimiento

1 Escuela de Ciencias de la Salud, Universidad Viña del Mar, Viña del Mar (Chile).

Autora de correspondencia: gpurrejolacontreras@gmail.com

2 Escuela de Kinesiología, Universidad Valparaíso (Chile).

3 Escuela de Odontología, Universidad de los Andes (Chile). 
comparado con el grupo control tanto en el promedio como en las evaluaciones parciales de cada unidad (t-test < 0.05). En el grupo experimental se registró un incremento del rendimiento entre el $59 \%$ y el $83 \%$ de los estudiantes. Adicionalmente, la mejora progresiva modificó la condición de reprobación a aprobación en un $27 \%$ de los estudiantes tras la intervención (Anova: $p$ < 0.05). Conclusión: la incorporación de evaluaciones de proceso parece ser una favorable herramienta para descomprimir las materias, aplicar lo aprendido, realizar seguimiento a las metodologías de estudio y mejorar el rendimiento de los estudiantes.

Palabras clave: evaluación proceso; rendimiento académico; anatomía; fisiología educación médica.

\section{Abstract}

Introduction: A current trend in higher education is to merge subjects together to create one integrated or joint subject. However, an important effect of this integration has been reduced student performance and an increase in subject failures. A possible way to combat this issue may be through a favorable relationship that has been described between the application of process evaluations as teaching and learning instruments in health sciences, especially for the subjects that have a large volume of content. The objective of this study was to assess the effect that this evaluation may have on students' performance in the integrated subject of anatomy and physiology in Health Sciences. Materials and methods: This was an experimental study consisting of 144 students from the School of Health Sciences who were studying the subject of Structure and Function and were distributed amongst two groups: control and experimental. A methodological intervention was designed and implemented to assess its effect on academic performance. Results: The intervention of this process in the experimental control group showed an improvement in students' performance compared to that of the control group both in the average and in the partial evaluations of each unit (t-test < 0.05). In the experimental group, there was an increase in performance between $59 \%$ and $83 \%$ of the total number of students. Additionally, the progressive improvement modified the condition of disapproval to that of approval in $27 \%$ of the students after the intervention, Anova $\mathrm{p}<0.05$. Conclusion: The incorporation of process evaluations seems to be a favorable tool to decompress subjects taught in schools, to apply what students have initially learned, to follow up on the study methodologies, and improve overall student performance.

Keywords: Students' perceptions; academic performance; learning; medical education.

\section{Resumo}

Introdução: uma tendência atual na educação superior é fusionar disciplinas e articular as disciplinas integradas; no entanto, há menor desempenho e um incremento da reprovação. Se tem descrito como favorável a aplicação de avaliações de processos como instrumentos de ensino e aprendizagem em ciências da saúde, critério pertinente de considerar em disciplinas de grande volume de conteúdo. Objetivo: O objetivo é valorar o efeito da avaliação do processo sobre uma disciplina integrada de anatomia e fisiologia em estudantes de ciências da saúde e sua relação com o desempenho. Materiais e métodos: estudo experimental com 144 estudantes da Escola de Ciências da Saúde na disciplina de Estrutura e Função, distribuídos em dois grupos: controle e experimental. Se desenhou e implementou uma intervenção metodológica para avaliar seu efeito no desempenho acadêmico. Resultados: a intervenção do processo no grupo controle experimental melhorou o desempenho comparado com o grupo controle tanto na média quanto nas avaliações parciais de cada unidade (t-test < 0.05 ). No grupo experimental se registrou um incremento do desempenho entre o 59\% e o $83 \%$ dos estudantes. Adicionalmente, a melhora progressiva modificou a condição de reprovação a aprovação em um 27\% dos estudantes após a intervenção (Anova: $\mathrm{p}$ < 0.05). Conclusão: a incorporação de avaliações de processo parece ser uma ferramenta favorável para descomprimir as matérias, aplicar o aprendido, realizar seguimento às metodologias de estudo e melhorar o rendimento dos estudantes.

Palavras-chave: avaliação processo; desempenho acadêmico; anatomia; fisiologia educação médica. 


\section{Introducción}

$F_{\text {demostrado ser un árido terreno tanto para docentes como para estudiantes, sobre todo }}^{\text {na la salud, tradicionalmente, la enseñanza de la anatomía y la fisiología ha }}$ por la extensión de sus contenidos, al igual que por los deficientes resultados obtenidos en términos de rendimiento y aprendizaje del alumnado, aspecto que aún hace considerar la asignatura de anatomía como crítica en términos de sus altos porcentajes de reprobación. A este escenario se suma el continuo proceso de cambio que ha enfrentado la docencia en el que, por ejemplo, son relevantes: el aumento en el número de estudiantes presentes en cada curso (principalmente por la demanda de ingreso a carreras del área de la salud), docentes con escasa experiencia en la enseñanza de estas materias y disminución o nula disponibilidad de materiales cadavéricos y el remplazo de estos por modelos anatómicos tridimensionales de más fácil acceso, manipulación y conservación (1).

Si bien el transcurso del tiempo ha traído disponibilidad de nuevas herramientas para la enseñanza, como medios digitales, también los estudiantes que afrontan estos cursos presentan características distintas para interactuar con los resultados y gestionar su aprendizaje. Sobre lo anterior, aunque las universidades han mostrado intentos por modernizar tanto la entrega como la evaluación de los contenidos mediante variadas estrategias metodológicas que incluyen recursos informáticos, la evidencia indica que solo un tercio de los estudiantes de las generaciones actuales — considerados "nativos digitales", "generación net” o "millennials”- los utiliza de forma complementaria para el estudio de la morfología-fisiología humana, y consideran que el desarrollo de trabajos del tipo estudio de casos y anatomía práctica contribuye mejor en la adquisición de habilidades como la aplicación, el análisis y la reflexión sobre los contenidos aprendidos (2).

La enseñanza de la anatomía-fisiología supone un reto docente adicional, que es contemplar la integración de dos disciplinas de gran exigencia para estudiantes de primer año que comienzan su formación profesional. Este proceso requiere validar dos tipos de resultados en el aprendizaje del alumnado: adquirir conocimientos de anatomía y fisiología básica y desarrollar habilidades que permitan aplicar lo aprendido. La interdependencia entre ambos dominios es clave para un adecuado rendimiento y éxito académico, y en este sentido las metodologías utilizadas para alcanzar estos objetivos resultan cruciales (3).

En la vida cotidiana, el desempeño de los estudiantes en asignaturas integradas de morfología-fisiología sigue presentando dificultades y bajos rendimientos. Algunos estudios han podido objetivar que una de las principales causas se encuentra vinculada con el proceso de evaluación tradicional que aún se practica ampliamente tanto para su enseñanza como para su posterior calificación, y un ejemplo de esto es que los propios estudiantes han declarado centrar su metodología en la utilización de recursos memorísticos (4). Esto, a su vez, limita el rendimiento debido al impacto que genera la monotonía en el estudiante, al abordar un 
contenido. Esto podría traducirse en una merma de las capacidades y las habilidades para la lectura, la interpretación de procesos y la resolución de casos mediante el análisis, así como falta de motivación para enfrentar un estudio dirigido y significativo.

Por otra parte, la enseñanza tradicional prioriza la repetición y el uso de la memoria, y si bien admite adquirir nociones, solo permite un aprendizaje superficial de difícil recuperación en la instancia de una evaluación en asignaturas que deben integrarse, como lo son la morfología y la fisiología, razón por la cual es necesario incluir herramientas que favorezcan un aprendizaje más centrado en la articulación de contenidos y en el análisis para obtener mejor rendimiento por parte de los estudiantes.

Intentando abordar esta problemática, Muñetón Gómez y Ortiz han hecho hincapié en que la intervención debe enfocarse en el apoyo de nuevos y diferentes recursos evaluativos que apunten a un entendimiento más acabado y profundo de una materia mediante el análisis (5). Tal aspecto acercará los contenidos al estudiante, y dejará de lado la rotulación y la estructuración de preguntas de reconocimiento e identificación de estructuras anatómicas (6).

En relación con lo descrito, la evaluación suele ser un aspecto sensible, ya que comúnmente los instrumentos se aplican una vez han concluido los tiempos de revisión de las materias, y entonces los estudiantes deben responder a la evaluación demostrando lo aprendido. Es posible que solo evaluar mediante instrumentos finales sea insuficiente para monitorear y realizar seguimiento del aprendizaje de los estudiantes, y a su vez estos carecen de una retroalimentación previa y continua que permita maximizar esfuerzos, modificar estrategias de estudios y focalizar correctamente los contenidos prioritarios y la relación asociativa entre ellos. Estudios específicos sobre la evaluación en morfología han sido enfáticos en mencionar que deben utilizarse diferentes tipos de evaluaciones y de recursos de enseñanza-aprendizaje, aplicarse tanto al inicio como al final de cada sesión, y todo recurso empleado debe ser calificado y retroalimentado al estudiante con el fin de evitar la permanencia de errores o identificación incorrecta de estructuras o procesos (7).

En este sentido, es relevante sugerir que la eventual aplicación de evaluaciones que validen el aprendizaje como resultado de un proceso activo del estudiante puede influir en la descompresión del volumen de contenidos y en el rendimiento de la evaluación final.

Es imperativo preguntar: ¿qué efectos sobre el rendimiento tiene la incorporación de evaluaciones sistemáticas de proceso? Este trabajo busca evaluar cuál es el efecto de la aplicación de evaluaciones de proceso en la asignatura integrada de anatomía y fisiología en carreras del área de la salud en el rendimiento académico. 


\section{Material y métodos}

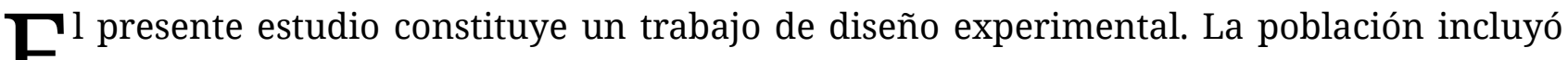
工a 165 estudiantes de primer año, pertenecientes a carreras de ciencias de la salud en la Universidad Viña del Mar (Chile). Los estudiantes considerados cursan la asignatura integrada de Estructura y Función, en la que se revisan estructuras de anatomía y temas de fisiología asociados a ellas. La asignatura es de carácter transversal para todas las carreras de la Escuela de Ciencias de la Salud, correspondiente al año 2019. La asignatura se imparte en el primer semestre del año en curso y tributa sobre ramas posteriores de cada carrera, orientadas en líneas de fisiopatología y fisiología asociada a sistemas corporales.

El criterio de inclusión fue que los estudiantes realizaran la asignatura por primera vez. Así, la población se separó en dos grupos: control $(n=80)$ y experimental $(n=85)$. Los criterios de exclusión fueron: aquellos estudiantes con inasistencias superiores al $75 \%$ de las sesiones y los que tramitaron durante el semestre solicitud de retiro temporal de sus estudios. El grupo control, al que no se le realizó intervención, estuvo constituido por 73 estudiantes pertenecientes a las carreras de Enfermería, Fonoaudiología y Kinesiología. El grupo experimental incluyó a 70 sujetos que corresponden a las carreras de Nutrición y Obstetricia. Por lo tanto, la muestra quedó finalmente conformada por 143 estudiantes.

La asignatura contempló la revisión de cuatro unidades temáticas durante el semestre, esto es: 1) introducción a la fisiología general y terminología anatómica, 2) sistema neuroendocrino, 3) sistema cardiorrespiratorio y 4) sistema digestivo-urogenital. La ponderación de cada unidad para tales efectos es equivalente y corresponde a un $25 \%$.

En el grupo control se llevó a cabo la revisión tradicional de los contenidos y, posteriormente, se aplicó un certamen para evaluar las materias correspondientes a cada unidad. En el grupo experimental se introdujo la intervención, que corresponde a la revisión de los contenidos, y se adicionó la resolución presencial de un taller grupal evaluado sumativamente. Las características de este taller consisten en resolución de casos y aplicación de contenidos inmediatamente después de haber finalizado la sesión de cátedra teórica tradicional. Los estudiantes se agrupan de a cinco por afinidad, realizan el taller en un tiempo de 50 minutos y posteriormente se entrega al docente. En la sesión siguiente se entrega una retroalimentación específica a cada grupo en la que se precisa la calificación obtenida, los errores y la forma de completar respuestas parciales o incorrectas. El taller se evalúa mediante una calificación obtenida a partir de una escala de exigencia correspondiente al $60 \%$. Se considera para la aprobación nota $\geq 4.0$, y reprobatoria una nota $\leq 3.9$.

Posteriormente, mediante un certamen se evaluaron los contenidos tratados en la unidad. Ambos grupos (control y experimental) mantuvieron la misma destinación horaria a cada clase presencial y calendario de evaluaciones correspondientes a los certámenes. La formulación de ambos instrumentos de evaluación para cada unidad se construyó a partir 
de una tabla de especificaciones y una matriz de preguntas. Ambos criterios delimitaron entonces su constructo y complejidad, siendo estas de equivalente dificultad y teniendo la misma estructura (igual número de preguntas de cada contenido, mismo tipo de preguntas e inferencias y análisis de casos para cada subtema).

La formulación de más de un instrumento de evaluación obedece a lo exigente de impartir una asignatura para un gran volumen de estudiantes. Esto dificulta la rendición de un mismo instrumento de evaluación por todos los estudiantes, al presentar dificultades horarias y disponibilidad de salas. Como reglamento institucional se respeta el horario para cada curso y asignatura, tanto en las sesiones programadas para clases como aquellas para evaluaciones. Por esto, como norma se utiliza la creación de dos instrumentos de evaluación - formulado y revisado en conjunto por todos los docentes, y de acuerdo con los lineamientos que especifica el programa de la asignatura y sus resultados de aprendizaje tanto en contenidos como en complejidad-. Sin perjuicio de lo anterior, y con el fin de asegurar un correcto instrumento de evaluación, su proceso de construcción lo lideraron docentes con formación en educación médica.

Una vez rendido cada certamen, se calificó con notas en una escala de 1 a 7, considerándose aprobatorio la nota $\geq 4.0$ y reprobatorio $\leq 3$.9. Se recopilaron los datos en una planilla de Excel para su posterior análisis.

La calificación presente en cada unidad en el grupo control se consiguió a partir de una ponderación del $100 \%$, obtenida mediante la nota del certamen rendido por los estudiantes; mientras que en el grupo experimental se alcanzó a través de una subponderación: promedio del taller (20\%) y certamen (80\%) (figura 1$)$.

Control

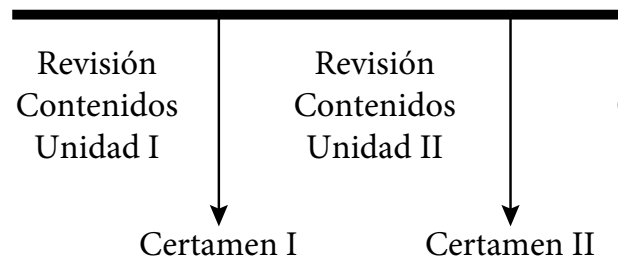

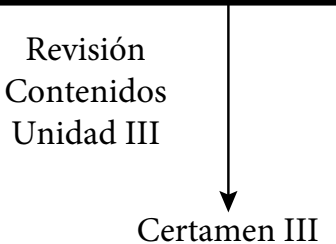

Certamen III

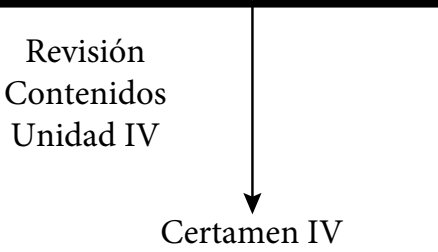

Experimental

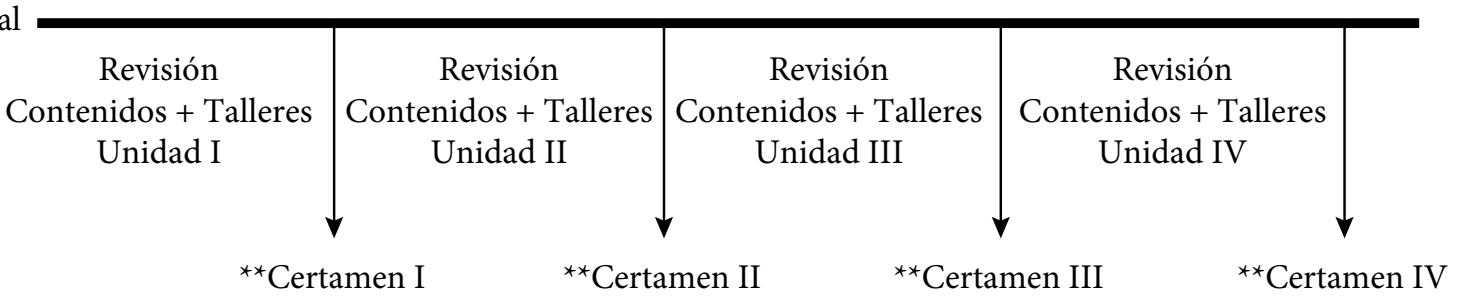

Figura 1. Diseño metodológico

Nota. En el grupo control, cada unidad está integrada por revisión de contenidos y su respectiva evaluación (certamen). En el grupo experimental, cada unidad está integrada por la revisión de contenidos, la resolución de talleres y sus respectivas evaluaciones (evaluación de taller y certamen).

* Metodología de talleres basada en análisis de casos y aprendizaje basado problemas en contexto de aplicación situacional para cada unidad.

** Nota certamen se obtiene mediante una ponderación entre certamen rendido (80\%) y promedio de talleres de la unidad respectiva (20\%). 
La formulación del taller incorporó el trabajo por medio de una metodología de análisis de casos y aprendizaje basado en problemas, construidos por docentes de la asignatura, los que además presentan formación en docencia. Se utilizaron videos, imágenes, mapas conceptuales, términos pareados y preguntas de desarrollo. El trabajo realizado por los estudiantes en el taller incluyó la resolución de cuatro problemas sobre la temática desarrollada en la clase expositiva presencial teórica del docente de cátedra. Cada problema es sobre una temática particular que contempla diferentes preguntas tanto en contenidos como en complejidad basados en los dos niveles iniciales de la pirámide de Miller (8). Esto es, reconocimiento de estructuras anatómicas y su ubicación topográfica mediante preguntas tipo i y asociación mediante inferencias de segundo orden entre estructuras anatómicas, funciones y descripción básica de procesos fisiológicos mediante preguntas tipo ir. Por lo tanto, la clase teórica del grupo experimental quedó conformada por la revisión del contenido hecha por el docente y, a continuación, la resolución del taller por parte de los estudiantes. Al finalizar la sesión, los estudiantes entregaron el taller resuelto a su docente. En la siguiente clase, este entregó la retroalimentación a cada grupo sobre el desempeño en el taller, resolución de dudas, identificación de errores y correcciones. Cada unidad presentó la resolución de tres talleres previo a la rendición del certamen correspondiente.

El análisis cuantitativo utilizó el programa Graph Pad 5 como paquete estadístico. Los datos del rendimiento académico obtenido de las cuatro unidades se expresan como la media 土 el error estándar (SEM) y se analizaron con un análisis de varianza (Anova de una vía), seguido de una prueba de comparación post hoc de Bonferroni, a menos que se indique lo contrario. La comparación del promedio del rendimiento entre el grupo control y el experimental se analizó mediante el prueba $t$ de Student. Para verificar los supuestos de Anova, se evaluó la normalidad de los datos utilizando el test Kolmogorov-Smirnov (K-S), y la igualdad de las varianzas, mediante el test de Levene. El nivel de significancia se estableció en $p<0.05$ $\left({ }^{*} p<0.05,{ }^{* *} p<0.001 \mathrm{y} * * * p<0.0001\right)$.

\section{Resultados}

\footnotetext{
A l comparar el rendimiento promedio de las cuatro unidades, se observó que el promedio del grupo experimental ( $4.3 \pm$ SEM) es significativamente superior, comparado con el grupo control (3.5 t-test < 0.05) (figura 2).
} 


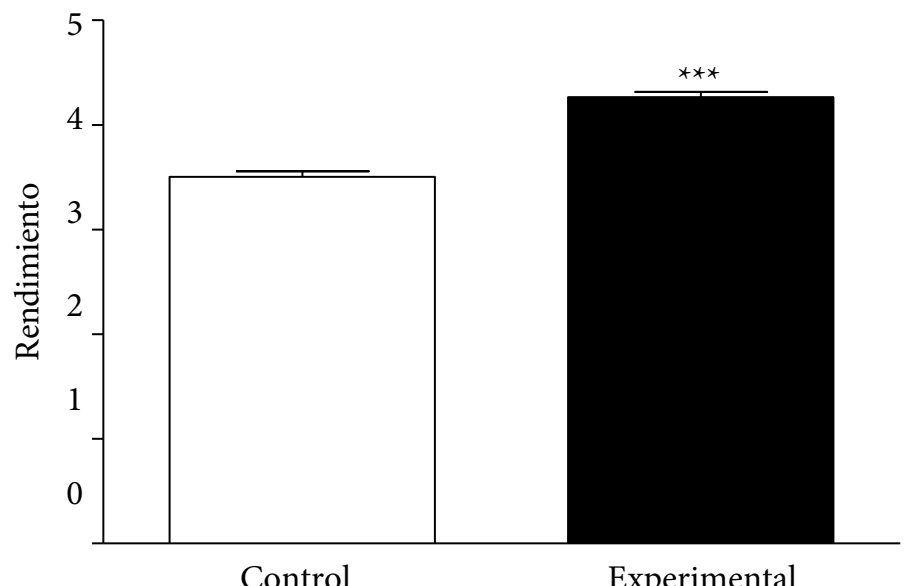

Figura 2. Comparación del rendimiento promedio entre ambos grupos: control y experimental

Nota. Los datos representan la media \pm SEM. ${ }^{* * *} \mathrm{p}<0.0001$

Adicionalmente, al comparar el rendimiento presente en cada certamen, el grupo control solo presenta aprobación en el certamen 1; mientras que el grupo experimental se visualiza en la totalidad de los certámenes (figura 3).

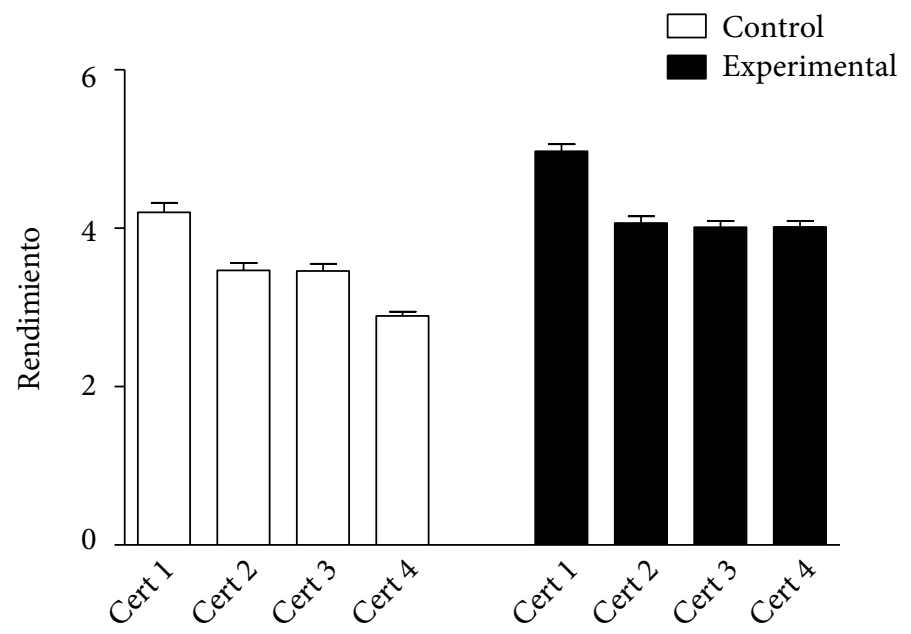

Figura 3. Rendimiento de las evaluaciones parciales

El grupo control ha obtenido solo un certamen superior al 4.0; mientras que en el grupo experimental la totalidad de las notas son superiores a 4.0. Los datos representan la media \pm SEM. Al analizar los cambios logrados en el grupo experimental se observó el porcentaje de estudiantes que obtiene un incremento en el rendimiento de su certamen respectivo a cada unidad mediante la realización del taller. Para el certamen 1 un $59 \%$ de los estudiantes reveló una mejora del rendimiento. A su vez, para los certámenes 2 y 3, un 82 \% y un $83 \%$, 
respectivamente, de los estudiantes muestra un incremento en el rendimiento. Por último, en el certamen 4, un $78 \%$ de los estudiantes muestra un aumento en sus calificaciones (figura 4).

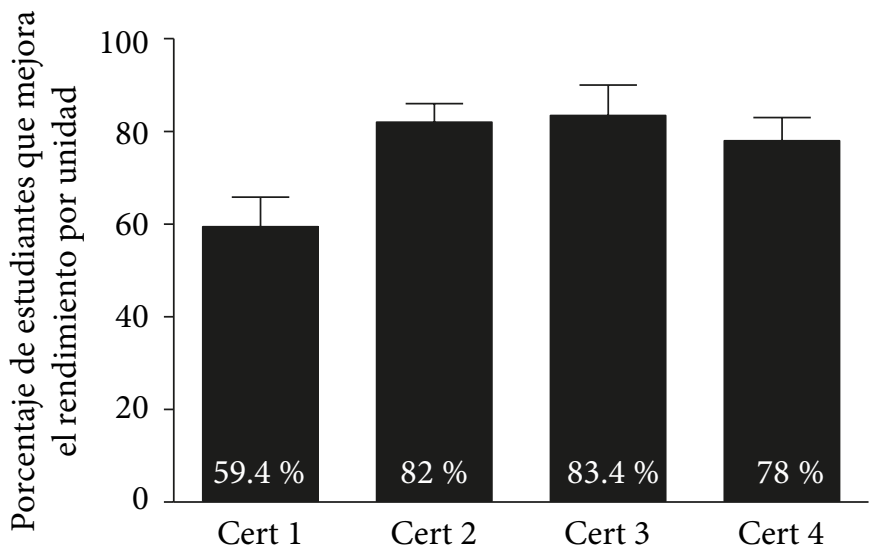

Figura 4. Porcentaje de estudiantes que experimentan un incremento del rendimiento en cada una de las unidades mediante la intervención del taller

Por último, al descomponer el rendimiento presentado por el grupo experimental, se registró el porcentaje de estudiantes que tras la realización del taller cambia su condición de reprobación a aprobación de la unidad en cada uno de los certámenes. En el certamen 1 corresponde solo a un $5 \%$. En el certamen 2 se observa una progresión en el que se visualiza un $18.5 \%$ de los estudiantes. Por último, en los certámenes 3 y 4 se incrementa a un 20.2\% y un $27 \%$ de los estudiantes evaluados (figura 5).

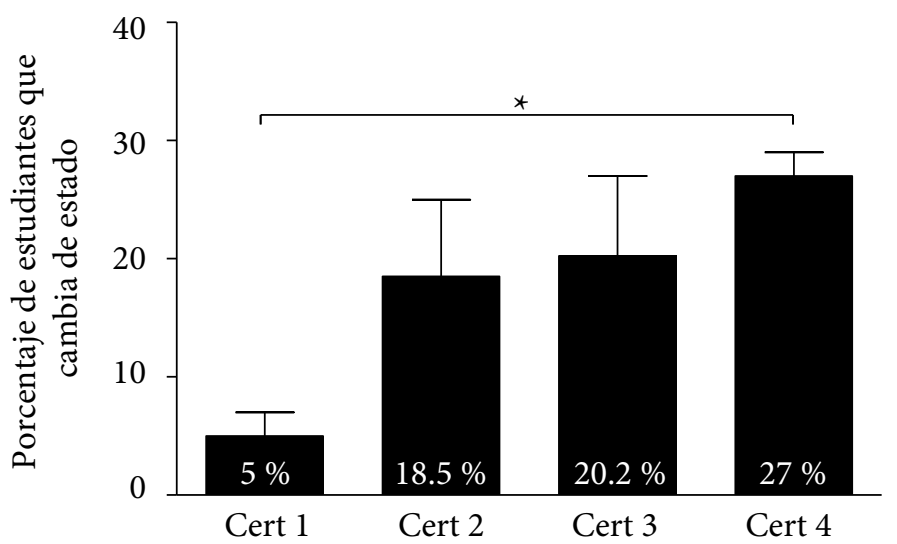

Figura 5. Porcentaje de estudiantes que modifica su condición de reprobación a aprobación en cada certamen

Nota. Los datos representan la media \pm sem. Existen diferencias significativas entre el cambio en la condición entre el certamen 4 y el certamen 1 (Anova de una vía $=\mathrm{p}<0.05)$.

Al analizar el rendimiento del grupo control en los diferentes certámenes, solo se observa una diferencia significativa en el certamen 1, a favor de la carrera de Fonoaudiología, en 
comparación con la carrera de Enfermería. En los certámenes 2, 3 y 4 no se observan diferencias entre las carreras; por tanto, se observó la misma tendencia en el rendimiento entre estos grupos. Al comparar el rendimiento obtenido entre las carreras que componen el grupo experimental de este trabajo, existió una diferencia significativa a favor de la carrera de Obstetricia para los certámenes 1 y 2 (figura 6).

(A)

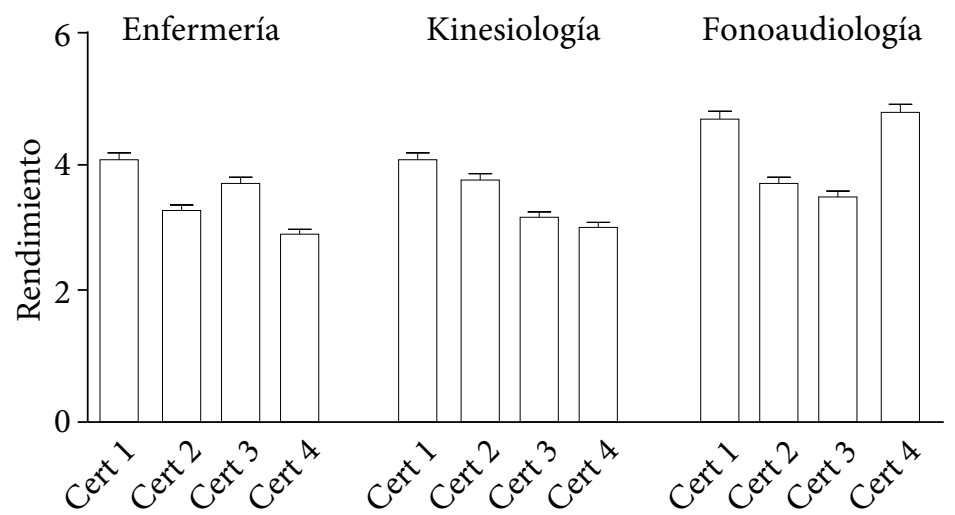

(B)

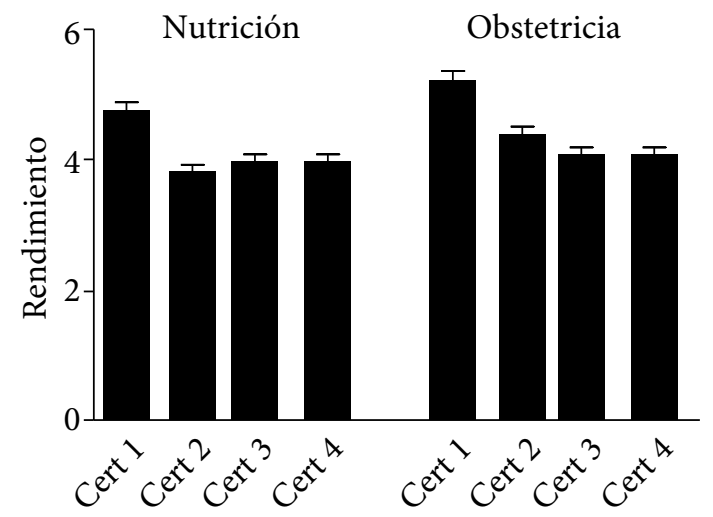

Figura 6. Comparación del rendimiento entre el grupo control (A) y el experimental (B) por carreras

Entre los grupos control (A) no existe diferencia significativa entre las carreras en el rendimiento de los certámenes, a excepción del certamen 1 de Fonoaudiología respecto al certamen 1 de Enfermería (Anova de una vía $=\mathrm{p}<0.05$, Bonferroni postest). En los grupos experimentales (B) existen diferencias significativas en los certámenes 1 y 2 entre las carreras de Obstetricia y Nutrición (t-test).

\section{Discusión}

T a variable de rendimiento en estructura y función ha revelado un incremento vinculado a Lla intervención en el proceso de evaluación y en la metodología utilizada para mejorar la aplicación de los contenidos como parte de un proceso de enseñanza y aprendizaje. A partir de ahí se descarta evaluar únicamente al final cada unidad revisada. Según la tendencia actual, se imparte la asignatura de anatomía o fisiología en carreras de salud durante el primer año de formación, lo que implica un desafío tanto para docentes como para estudiantes, ya que estos promedian edades cercanas a los 20, etapa en la que la inmadurez y las falencias presentes, como carecer de metodologías de estudio, pueden asomarse como factores de riesgo asociados al fracaso académico en la aproximación inicial en cada una de las carreras del área de salud (9). 
Sobre lo mencionado, es importante destacar que actuales directrices en ciencias de la salud, y desde los cambios promovidos a partir de la Declaración de Bolonia y el Espacio Europeo de Educación Superior, se han propuesto avanzar en la generación de un nuevo modelo docente y en el aprendizaje multiprofesional, creando asignaturas integradas a lo largo de la formación disciplinar de cada carrera, a fin de basar la docencia en aprendizaje basado en problemas y facilitar la asociación de disciplinas relacionadas en su solución (10). Sin embargo, este proceso de transformación debe atender la complejidad de integrar asignaturas que de forma simultánea incluyen gran volumen de contenidos, aspectos que dificultan un adecuado tránsito y rendimiento en los estudiantes cuando estos carecen de formación universitaria previa.

La incorporación de estrategias que mitiguen ese riesgo y afronten de mejor forma el aprendizaje, al facilitarle al estudiante la aplicación y resolución de problemas de manera supervisada, promueve la instrucción y la experiencia, otorgándole mayor diligencia; además, permite ponderar y amortiguar los temores asociados al fracaso de una eventual evaluación única y final. En este sentido, Castillo, et al. recogen en su estudio la percepción de los propios estudiantes y docentes sobre el uso de metodologías que abordan e integran disciplinas diferentes, pero a la vez complementarias (11). Los criterios mejor valorados son: una mejor comprensión y relación de las materias, un mayor dinamismo en las clases y la mejora de nexos entre estudiantes y docentes a través de un trabajo motivador y enriquecedor.

La mejora en el rendimiento obtenido en el grupo experimental puede obedecer a los principios valorados en la sincronía entre la revisión de contenidos, la resolución de talleres calificados y la evaluación final. Asimismo, una evaluación debe contemplar aspectos o criterios que construyan adecuadamente un proceso y predigan un mejor desempeño, ya sea en instancias formativas o en sumativas, entre las cuales se encuentran: método de enseñanza utilizado y practicado, contenido del objeto específico que se va a evaluar y formato de preguntas o problemas. Por otra parte, la incorporación de los talleres permitió, a su vez, validar las funciones de una evaluación de proceso, la que rescata aspectos como: retroalimentar, educar, instruir y establecer un punto de comprobación y control de lo aprendido (12).

A modo de análisis posterior y reflexión, una limitante de esta investigación es no haber contado con una caracterización específica de la muestra estudiada, aspecto que pudo de alguna forma diferenciar a los estudiantes según sus cualidades, fortalezas y debilidades individuales como grupales. Si bien la aproximación al éxito académico es multifactorial, algunos autores han descrito que la influencia de variables individuales de los estudiantes como características socioeconómicas, elementos de naturaleza académica y psicológicas pueden conjugarse y promover o impactar negativamente en el rendimiento, aspectos que podrían estar presentes en la muestra estudiada y que podrían repercutir en la diferencia de rendimiento entre algunas carreras (13); sin embargo, no se evaluaron tales aspectos. 
Aunque el objetivo de este trabajo no fue analizar las diferencias entre estudiantes de diferentes carreras, sino evaluar la efectividad de una herramienta metodológica sobre el rendimiento, hemos podido constatar que sí existen diferencias. No obstante, la tendencia de rendimiento es similar, razón por la que se combinaron estudiantes de distintas carreras para formular el grupo control y el grupo experimental.

En su contraparte, una fortaleza relevante de esta investigación es que considera las diferencias presentes en el grupo experimental entre las calificaciones de la primera y la cuarta unidad. Este trabajo ha permitido detectar el efecto en el rendimiento del estudiante el entender una metodología para posteriormente generar más y mejor aprendizaje, lo que fue demostrado en la progresión de las calificaciones $(14,15)$.

Esto se debe en mayor medida a la ventaja que ofrece un proceso de enseñanza y de evaluación permanente que sigue y monitorea los objetivos del curso y los resultados de aprendizaje esperados. En conjunto, proporciona información que brinda elementos para la realización de ajustes y retroalimentar al estudiante para mejorar su proceso (16).

Así, es posible considerar que la herramientra utilizada fortaleció tres conceptos relevantes en educación médica: primero, la incorporación de talleres subponderados para cada unidad de trabajo ha sido positiva en el rendimiento obtenido mediante la ganancia de dos fortalezas: la reducción de los tiempos de estudio previo al certamen y el andamiaje sistemático de retroalimentación, que favorece un desarrollo óptimo de los contenidos que específicamente cada grupo de estudiantes debía reforzar. Ambos conceptos resultan cruciales en la descripción de una evaluación auténtica $(17,18)$.

Segundo, lo constituyen los elementos que permiten evidenciar cómo la estrategia metodológica promueve el aprendizaje significativo y de esto se desprende que un mejor rendimiento se encuentra precedido de un estudio menos memorístico, pero con mayor capacidad de reflexión y análisis. La resolución de talleres para esta asignatura integrada buscó conceptualizar el conocimiento en escenarios de aplicación. Estudios similares han demostrado que estas metodologías activan el aprendizaje de un mayor número de relaciones a partir de unidades sencillas de información, entre ellas la morfología y las funciones de las estructuras presentes en un sistema fisiológico $(19,20)$.

Por último, ¿qué rol desempeña lo mencionado en términos curriculares? La organización del currículo es la columna vertebral de un modelo de enseñanza y requiere una correcta gestión que derive en acciones que delimiten la selección de contenidos, ubicación en un programa educativo y en la malla curricular, metodologías que se vayan a utilizar y diseño de sistemas de evaluación coherentes y pertinentes (21). En este trabajo, si bien la asignatura elegida se imparte de forma transversal para todas las carreras presentes en la Escuela de Salud, se consideró una estructura que atendiera las necesidades de cada carrera participante en a) integración de ciencias básicas, b) intervención de contenidos que tributan a asignaturas siguientes como fisiopatología, c) complejidad adecuada a estudiantes de primer año y d) 
incorporación de actividades que promuevan la mejora de habilidades como la búsqueda de información en textos o internet como complemento a lo revisado por los docentes en clases teóricas presenciales. Los aspectos señalados e incluidos se han descrito como pilares en la incorporación de metodologías que han resultado ser exitosas académicamente, pero también como herramienta de ayuda en la transición estudiantil, aquella que también provee al estudiante de una mayor autoeficacia mediante la confianza, la autonomía y el compromiso con sus estudios $(22,23)$.

Producto de los resultados arrojados en este estudio, la estrategia didáctica se incorporó para el resto de las carreras no participantes en esta instancia, y se integró como parte de las metodologías que se deben utilizar en las asignaturas posteriores a Estructura y Función, según cada malla curricular.

En este sentido, es crucial promover el uso de estrategias que utilicen la evaluación en todas sus dimensiones como recurso de aprendizaje y no solo como instancia de calificación. Así, por ejemplo, las evaluaciones de proceso o intermedias pueden constituir una herramienta que facilita incorporar actividades que soliciten distintas habilidades de aprendizaje, resolución de problemas y guiar aprendizaje mediante búsquedas en la web.

\section{Contribución de los autores}

Todos los autores contribuyeron en las distintas fases del estudio y en la redacción de este Gabriela Urrejola Contreras: concepción y diseño del trabajo, recolección y obtención de los resultados, análisis e interpretación de datos, redacción y revisión crítica del artículo y aprobación de su versión final.

Paulina Tenore Venegas y Sebastián Elgueta Perinni: aporte de material de estudio y asesoría administrativa.

Miguel Pérez Lizama: análisis e interpretación de datos, asesoría estadística, revisión crítica y aprobación final del artículo.

Paula Araya Herrera: revisión crítica, aprobación de la versión final, asesoría estadística.

Pía Daniela Campos Reinoso: redacción y revisión crítica del artículo, y aprobación de la versión final del artículo.

\section{Conflicto de intereses}

Ninguno declarado. 
Talleres y evaluaciones de proceso: una herramienta que mejora el rendimiento en asignaturas integradas

\section{Referencias}

1. Inzunza HO, Salgado AG. Evaluaciones prácticas objetivadas en anatomía: diferencias de rendimiento en preguntas realizadas en modelos, preparaciones anatómicas y cadáveres. Int J Morphol. 2011;29(2):490-5. Doi: 10.4067/S0717-95022011000200031

2. Rodríguez-Herrera R, Losardo RJ, Binvignat O. La anatomía humana como disciplina indispensable en la seguridad de los pacientes. Int J Morphol. 2019;37(1):241-50. Doi: 10.4067/S0717-95022019000100241

3. Mompeó-Corredera B. Metodologías y materiales para el aprendizaje de la anatomía humana: percepciones de los estudiantes de medicina 'nativos digitales'. FEM. 2014;17(2):6. Doi: 10.4321/S2014-98322014000200007

4. Cárdenas OC, Briceño MO. Rendimiento académico en Anatomía Humana en estudiantes de kinesiología: aproximación a sus causas y efectos. Educación Médica Superior. 2018;32(2):12.

5. Muñetón Gómez CA, Ortiz JA. Plastinación: un instrumento complementario para el desarrollo del proceso enseñanza-aprendizaje de la anatomía. Rev Med Vet. 2012;(23):1117. Doi: $10.19052 / \mathrm{mv} .79$

6. Silva Ortiz SR, Torres Merchán NY. Significado del aprendizaje y la enseñanza de la anatomía: contribuciones desde las percepciones de los estudiantes. Zona Próxima [Internet]. 2012;(17). Disponible en: http://rcientificas.uninorte.edu.co/index.php/zona/ article/viewArticle/3310/214421442213

7. Gal-Iglesias B, de Busturia-Berrade I, Garrido-Astray MC. Nuevas metodologías docentes aplicadas al estudio de la fisiología y la anatomía: estudio comparativo con el método tradicional. Educ Méd [Internet]. 2009 [citado 2019 nov 8];12(2):117-24. Disponible en: http://scielo.isciii.es/scielo.php?script=sci_abstract\&pid=S1575-18132009000300008

8. Pinilla-Roa AE. Evaluación de competencias profesionales en salud. Rev Fac Med. 2013;61(1):53-70.

9. García-Hernández F. Evaluación del aprendizaje práctico de la anatomía humana para odontología en la Universidad de Antofagasta, Chile. Int J Morphol. 2003;21:43-7.

10. Coll J, Bernabeu D, Cervantes C, Nolla M, Muniesa JM, Tor J, et al. Un modelo de aprendizaje multiprofesional en ciencias de la salud: innovación docente como respuesta a las necesidades emergentes de nuestra sociedad. Educ Méd [Internet]. 2010 [citado 2019 nov 8];13 (supl. 1). Disponible en: http://scielo.isciii.es/scielo.php?script=sci_arttext\&pi$\mathrm{d}=$ S1575-18132010000500004

11. Castillo BC, Arriaga A, Bregains LE, Gómez ME, Flores VY, Sanz AV, et al. Enseñanza integrada de anatomía e histología en la Facultad de Odontología de la Universidad Nacional de Córdoba, Argentina. FEM. 2015;18(4):253-9. Doi: 10.4321/S2014-98322015000500006

12. Muntinga ME, Krajenbrink VQE, Peerdeman SM, Croiset G, Verdonk P. Toward diversity-responsive medical education: taking an intersectionality-based approach to a curriculum evaluation. Adv Health Sci Educ. 2016;21(3):541-59. Doi: 10.1007/s10459-015-9650-9

13. Brito-Jiménez IT, Palacio-Sañudo J. Calidad de vida, desempeño académico y variables sociodemográficas en estudiantes universitarios de Santa Marta-Colombia. Duazary. 27 de julio de 2016;13(2):133. Doi: 10.21676/2389783X.1719 
14. Pérez Sánchez AM, Leticia María BA. La evaluación como actividad orientada a la transformación de los procesos formativos. Educación Médica Superior. 2004;18:1-1.

15. Santelices L, Williams C, Soto M, Dougnac A. Efecto del enfoque de autorregulación del aprendizaje en la enseñanza de conceptos científicos en estudiantes universitarios en ciencias de la salud. Rev Méd Chile. 2014;142(3):375-81.

16. Flores FF, Michel NC, González AM. Evaluación del aprendizaje en la educación médica. Rev Fac Med (Méx) [Internet]. 2012;55(3):42-8. Disponible en: http://www.scielo.org.mx/ scielo.php?script=sci_arttext\&pid=S0026-17422012000300008

17. De Jong N, Savin-Baden M, Cunningham AM, Verstegen DML. Blended learning in health education: three case studies. Perspect Med Educ. 2014;3(4):278-88. Doi: 10.1007/s40037014-0108-1

18. Harrison CJ, Könings KD, Dannefer EF, Schuwirth LWT, Wass V, van der Vleuten CPM. Factors influencing students' receptivity to formative feedback emerging from different assessment cultures. Perspect Med Educ. 2016;5(5):276-84.

19. Cavanagh A, Vanstone M, Ritz S. Problems of problem-based learning: Towards transformative critical pedagogy in medical education. Perspect Med Educ. 2019;8(1):38-42.

20. Greville WJ, Dymond S, Newton PM. The student experience of applied equivalence-based instruction for neuroanatomy teaching. J Educ Eval Health Prof. 2016;13:32. Doi: 10.3352/ jeehp.2016.13.32

21. Ramírez Montes OS, Navarro Vargas JR. El aprendizaje basado en problemas y su utilidad en el desarrollo curricular en las ciencias de la salud. Rev Fac Med. 2015;63(2):325-30.

22. Chan AYC. Approaches of anatomy teaching for seriously resource-deprived countries: a literature review. Educ Health. 2019;32(2):62-74. Doi: 10.4103/efh.EfH_272_17

23. Mendoza-Espinosa H, Méndez-López JF, Torruco-García U. Aprendizaje basado en problemas (ABP) en educación médica: sugerencias para ser un tutor efectivo. Investigación Educ Médica [internet].2012;1(4):235-7. Disponible en: http://www.scielo.org.mx/scielo. php?script=sci_abstract\&pid=S2007-50572012000400011\&lng=es\&nrm=iso 